\title{
SPACECRAFT THERMAL DESIGN WITH THE GENERALIZED EXTREMAL OPTIMIZATION ALGORITHM
}

\author{
Roberto L. Galski, Fabiano L. de Sousa, Fernando M. Ramos and Issamu Muraoka \\ Instituto Nacional de Pesquisas Espaciais (INPE) \\ Av. dos Astronautas, 1758 - 12227-010 - São José dos Campos - SP - Brasil \\ galski@ccs.inpe.br,fabiano@dem.inpe.br,fernando@lac.inpe.br, issamu@dem.inpe.br
}

\begin{abstract}
This paper describes an application of the Generalized Extremal Optimization (GEO) algorithm to the inverse design of a spacecraft thermal control system. GEO is a recently proposed global search meta-heuristic [1], [2], [3] based on a model of natural evolution ${ }^{[4]}$, and specially devised to be used in complex optimization problems ${ }^{[5]}$. Easy to implement, GEO has only one free parameter to adjust, does not make use of derivatives and can be applied to constrained or unconstrained problems, non-convex or even disjoint design spaces, with any combination of continuous, discrete or integer variables. The application reported here concerns the optimum design of a simplified configuration of the Brazilian Multimission Platform (in Portuguese, Plataforma MultiMissão, PMM) thermal control subsystem, comprising five radiators and one battery heater. The PMM is a multi-purpose space platform to be used in different types of missions such as Earth observation, scientific or meteorological data collecting. The design procedure is tackled as a multi-objective optimization problem, considering two critical, operational hot and cold cases. The results indicate the existence of non-intuitive, new and more efficient design solutions.
\end{abstract}

\section{LIST OF SYMBOLS}

$\mathrm{F}(\mathbf{X})=$ Objective function;

$\mathbf{G}_{\mathrm{i}, \mathrm{j}}=$ Conductive conductance between panels $\mathrm{i}$ and $\mathrm{j}$;

$\mathrm{k} \quad=$ Ranking position for a given bit;

$\mathrm{L}=$ Total number of bits used to codify the variables using GEO;

$\mathrm{Lj}=$ Number of bits used to codify the $\mathrm{j}$-th variable using $\mathrm{GEO}_{\mathrm{var}}$;

$\mathrm{N}$ = Number of design variables;

$\mathrm{P}_{\mathrm{k}} \quad=$ Mutation probability for the k-th ranked bit;

$\mathbf{q}_{\text {ext }}[1: 5]=$ External radiation flux incident on panels 1 to 5;

$\mathbf{Q}_{\text {int }}[1: 5]=$ Heat generated by the equipments on panels 1 to 5 ;

$\mathbf{R}_{\mathrm{i}, \mathrm{j}} \quad=$ Radiative conductance between panels $\mathrm{i}$ and $\mathrm{j} ;$
$\mathrm{T}_{\propto} \quad=$ Deep space absolute temperature;

$\mathbf{T}[1: 6]=$ Vector containing the absolute temperatures on panels 1 to 6 ;

$\mathbf{T}_{\mathrm{cc}} \quad=$ Vector containing the Cold Case (CC) temperatures on panels 1 to 6 ;

$\mathbf{T}_{\text {снс }}=$ Vector containing the Cold and Hot Case (CHC) temperatures on panels 1 to 6;

$\mathbf{T}_{\mathbf{H C}}=$ Vector containing the Hot Case (HC) temperatures on panels 1 to 6 ;

$\mathbf{T}_{\text {MIN }}=$ Vector containing the lower limits for the temperatures on panels 1 to 6 ;

$\mathbf{T}_{\mathrm{MAX}}=$ Vector containing the upper limits for the temperatures on panels 1 to 6 ;

$\mathbf{T}_{\mathrm{T}} \quad=$ Vector containing the target temperatures for panels 1 to 6 ;

$\mathbf{X}[1: 5]=$ Design variables 1 to 5 . (radiator areas of panels 1 to 5 , respectively);

$\mathbf{X}[6]=$ Sixth design variable. (battery heater power);

$\mathbf{X}_{\mathrm{MIN}}=$ Vector containing the lower limits for the design variables;

$\mathbf{X}_{\mathrm{MAX}}=$ Vector with the upper limits for the design variables;

$\alpha=$ Absortivity of the radiator coating;

$\varepsilon \quad=$ Emissivity of the radiator coating;

$\tau=$ Free parameter of the GEO and $\mathrm{GEO}_{v x}$ algorithms;

$\tau^{*} \quad=\tau$ optimum value for a given $\mathrm{F}(\mathbf{X})$;

\section{INTRODUCTION}

Many numeric techniques have been developed to address optimization problems in

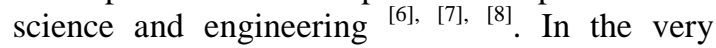
beginning, the main concern was to find a solution, by starting from a given point (or a set of points) in the search space and using information from the vicinities of that point(s). In that sense, a solution was a point where no additional improvement could be made on the defined objective function, because all points on the neighborhood of the solution point led to a worse value of the objective function. Nowadays, 
those solutions are called local solutions, local extremes, or suboptimal solutions of the optimization problem, because they are totally dependent on the location of the starting point(s) and there is no guarantee of any kind they are, in fact, the optimal (global) solutions. These earlier methods are called local search optimization methods. There is no doubt about the usefulness of such methods, since, by any means, a locally improved solution is better than a non-improved one. Unfortunately, it is also known, that many, perhaps the majority, of the scientific and engineering optimization problems are nonlinear, multimodal and most of them subject to several restrictions on the variables ${ }^{[9]}$. This fact, plus the increasing computational power of nowadays computers, have increased the interest for global optimization methods ${ }^{[7]}$, where the main concern is not to find only a locally improved solution, but a globally improved one, that is, the optimal solution. In the last twenty years, a considerable number of global methods have been developed. Most of them are based on natural phenomena analogies, trying to copy the efficiency and simplicity of observed self-optimized processes in nature. Algorithms based on the evolution of species ${ }^{[10],[11]}$, on the annealing of metals ${ }^{[12]}$, on the functioning of the brain ${ }^{[13]}$, on the immune system ${ }^{[14]}$ and even on the social behavior of ants [15] have been developed and used to get optimized solutions for many science and engineering problems. Among them, perhaps the most commonly used are Simulated Annealing (SA) ${ }^{[12]}$, Genetic Algorithms (GAs) ${ }^{[10]}$ and their derivatives. More recently, it has been seen an increasingly number of references formulating and dealing with optimization problems as multiobjective ones ${ }^{[16]}$. Despite of being computationally costly, a multiobjective approach brings a complete set of optimal compromise solutions to the problem, the so-called Pareto ${ }^{[17]}$ Frontier, and this is a powerful information to the designer.

The Generalized Extremal Optimization algorithm (GEO) ${ }^{[1],[2], ~[3]}$, like SA and GA, is a stochastic algorithm, but unlikely these ones, it has only one free parameter $(\tau)$ to be set, instead of three or more. In this paper, the GEO algorithm is used to found optimized radiator areas for the PMM spacecraft considering two critical hot and cold operational cases. In the following, both GEO and PMM are described with further details.

\section{THE GEO ALGORITHM}

The Generalized Extremal Optimization (GEO) algorithm is a global search meta-heuristic [1], [2], [3], based on a model of natural evolution ${ }^{[4]}$, and specially devised to be used in complex optimization problems ${ }^{[5]}$. It has its fundaments on the Self-Organized Criticality (SOC) theory, which has been used to explain the power law signatures that emerge from many complex systems ${ }^{[18]}$.

A flowchart for GEO and its variant $\mathrm{GEO}_{\text {var }}$ (see next paragraph) is presented in the Figure 1. In the flowchart, $F(X)$ is the objective function, $k$ is the ranking value of the bit and $L_{j}$ is the number of bits of the design variable " $\mathrm{j}$ ".

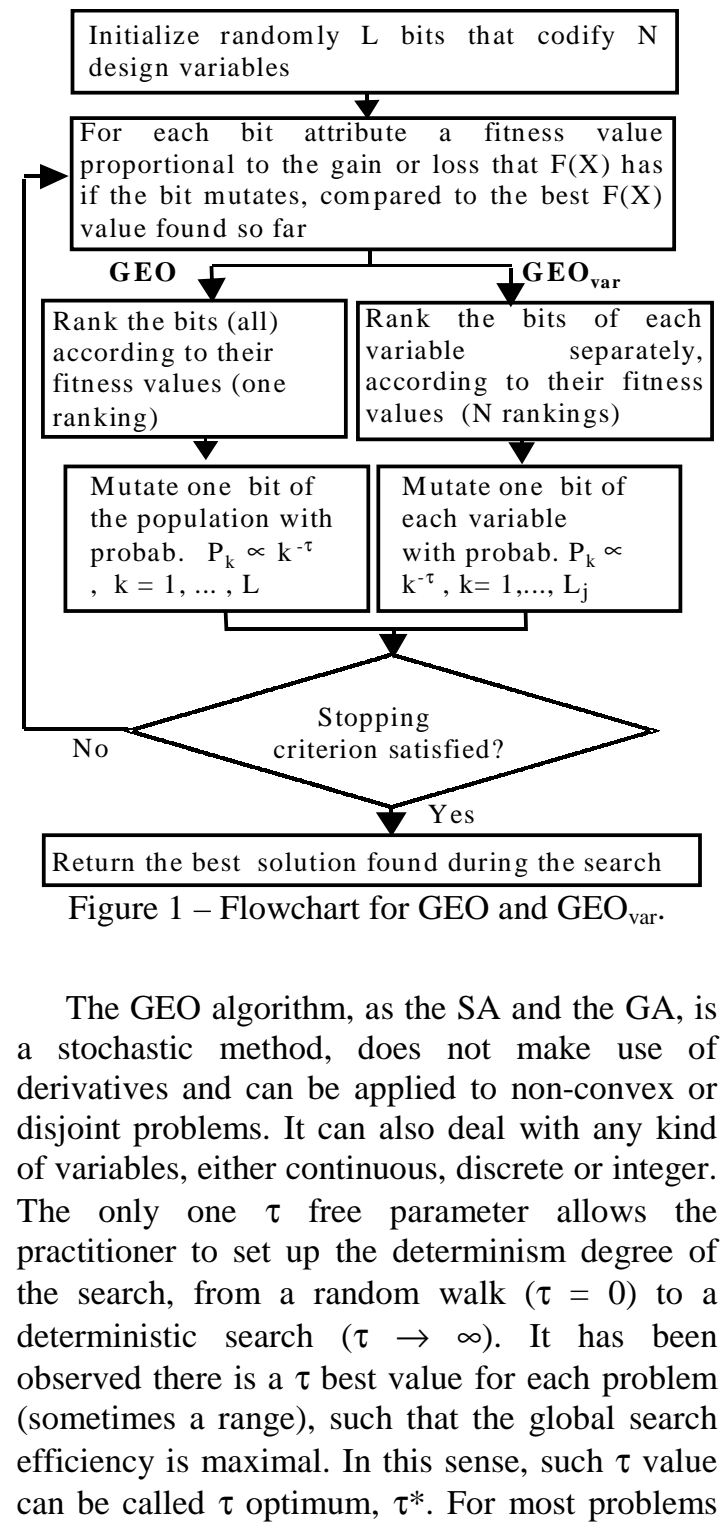


tackled with GEO, what has been seen is that $\tau^{*}$ remains in the 1 to 5 interval. GEO has one variant, called $\mathrm{GEO}_{\mathrm{var}}$. A detailed explanation of them, including application examples, can be found in ${ }^{[2], ~[3]}$. Here, both GEO and $\mathrm{GEO}_{\text {var }}$ will be used for the addressed optimization problem.

\section{THE PMM SPACECRAFT}

The Multi-Mission Platform (in Portuguese, Plataforma Multi-Missão - PMM) is a multipurpose space platform to be used in different types of missions such as Earth observation, scientific or meteorological. The PMM is a concept of satellite architecture that consists of assembling in a platform all the necessary equipment essential to the satellite, independent of the orbit or pointing mode. In this kind of architecture, there is a physical separation between platform and payload modules, which can be developed, constructed and tested separately, before the integration and final test. There is also the advantage of reuse of the platform project and reduction of the cost for the development of new satellites.

Table 1. Multi-mission Platform characteristics

\begin{tabular}{|c|c|}
\hline Characteristics & Value \\
\hline Dimensions & $1 \mathrm{~m} \times 1 \mathrm{~m} \times 1 \mathrm{~m}$ \\
\hline Mass & $250 \mathrm{~kg}$ \\
\hline Power Consumption & $150 \mathrm{~W}$ \\
\hline Payload Power Supply & $175 \mathrm{~W}$ \\
\hline Orbit Inclinations & $\begin{array}{c}\text { near equatorial }\left(\mathrm{i}<15^{\circ}\right) \\
\text { and sun-synchronous }\end{array}$ \\
\hline Orbit altitudes & $600 \mathrm{~km}$ to $1200 \mathrm{~km}$ \\
\hline Attitude & $\begin{array}{l}\text { Earth, Sun or inertial } \\
\text { pointing }\end{array}$ \\
\hline Maneuver capabilities & $\Delta \mathrm{V}=150 \mathrm{~m} / \mathrm{s}$ \\
\hline Data storage capability & 2 Gbits \\
\hline
\end{tabular}

Figures 2 and 3 illustrate the PMM configuration.

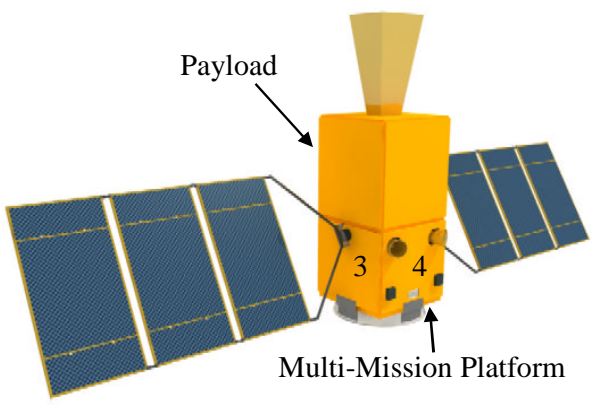

Figure 2 - Schematics of a payload attached to the Multi-Mission Platform PMM.

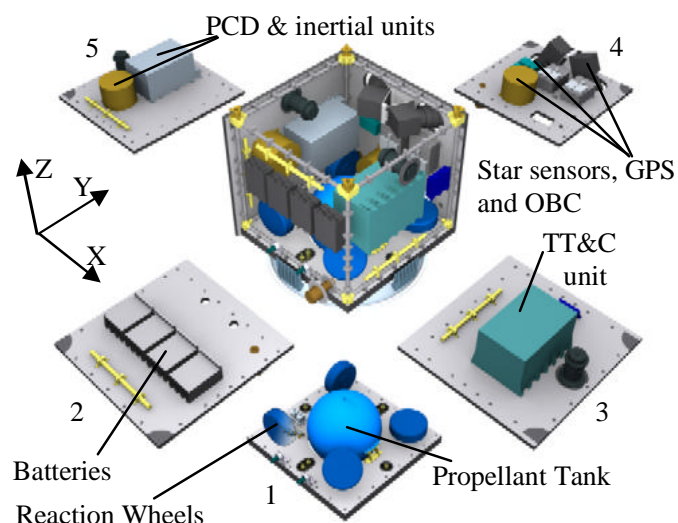

Figure 3 - Simplified view of the PMM and its panels 1 to 5 showing some internal devices.

The main goal of the thermal design of a spacecraft is to keep the temperature of the elements of the vehicle within their required ranges. One of the most important issues the satellite thermal engineer has to address is the definition of the size and position of the radiators. Radiators are areas of the satellite covered with high emissivity coating, so that they can reject heat to space to keep the temperature of the spacecraft equipment within their required design range during periods of high heat dissipation in the electronic equipment and/or high external thermal loads. On the other hand, these areas must not be exceedingly large so that, during periods of low heat loads, the temperatures do not go below the allowed minimum. In the case of the PMM, radiators can be positioned in 5 of the 6 sides of the platform body, since the top side does not "see" the space due to the payload mounted on it (see Figure 2).

In satellite thermal design two critical situations are usually identified, where minimum and maximum temperatures are expected to occur: i) The Cold Case (CC), when the external heat loads (solar radiation, earth radiation and albedo) are minimal, the satellite is operating with the lowest heat dissipation in the electronic equipment, and the thermal optical properties of its coatings are nondegraded and; ii) The Hot Case (HC), when the external heat irradiation is maximal, the satellite is in operational mode with the highest heat dissipation and the optical properties of the coatings are degraded. The thermal design shall manage the heat flow in a way that, in both situations, the temperatures of all elements remain within the required range of temperature. There are many variables, such as the size of radiators, that affect the 
temperature distribution, and the thermal engineer has to find a combination of these variables to reach a satisfactory design. This task needs a lot of simulations and analysis, considering the large numbers of variables involved.

In equipment where a strict control of the temperature is required, such as in the batteries of the PMM (panel 2), heaters are frequently used to warm the equipment during the $\mathrm{CC}$. On the other hand, as the electrical power supply is very limited in a satellite, the power spent on the heaters must be the least possible.

In this study a specific mission of the PMM was analyzed, defined by equatorial orbit with altitude of $600 \mathrm{~km}$ and having the battery panel always pointing to Earth along the orbit.

\section{FORMULATION OF THE INVERSE DESIGN PROBLEM}

The objective of the application of GEO to the PMM thermal design is to reduce the time spent by the engineer to find, not only a satisfactory but also an optimum (or quasi-optimum) design, a process that is done traditionally "manually", with the thermal designer running multiple analysis cases. In this paper, a design is a set comprising five radiator areas and one battery heater power.

The optimization problem consists in, by varying the area of the radiators on each one of the 5 panels, minimize the difference between the temperatures calculated for the $\mathrm{CC}$ and $\mathrm{HC}$ and given target temperatures for each panel, and at the same time minimize the battery heater power dissipation.

As the satellite is still in the early stages of development, a simplified numerical model was made only considering the six PMM sides, with the equipment simulated as heat sources over their respective panels. Panels 1 to 5 exchange heat with each other, by conduction and/or radiation, and with the space environment, by radiation, through the radiators placed on them. The top panel (not shown in Figure 3) makes the interface with the payload and is thermally isolated from it, but exchange heat with the other panels of the PMM.

Using the lumped parameter representation $^{[19]}$ and assuming steady state condition with orbit average heat loads, the heat balance at each one of the six panels leads to the following equations:

$$
\begin{aligned}
& \sum_{\mathrm{j}=1}^{6} \mathbf{G}_{1, \mathrm{j}}(\mathbf{T}[1]-\mathbf{T}[\mathrm{j}])+\sum_{\mathrm{j}=1}^{6} \mathbf{R}_{1, \mathrm{j}}\left(\mathbf{T}[1]^{4}-\mathbf{T}[\mathrm{j}]^{4}\right)+ \\
& +\varepsilon \mathbf{X}[1]\left(\mathbf{T}[1]^{4}-\mathrm{T}_{\infty}^{4}\right)=\mathbf{Q}_{\mathrm{int}}[1]+\alpha \mathbf{X}[1] \mathbf{q}_{\mathrm{ext}}[1]
\end{aligned}
$$

$$
\begin{aligned}
& \sum_{j=1}^{6} \mathbf{G}_{2, j}(\mathbf{T}[2]-\mathbf{T}[\mathbf{j}])+\sum_{\mathrm{j}=1}^{6} \mathbf{R}_{2, \mathrm{j}}\left(\mathbf{T}[2]^{4}-\mathbf{T}[\mathbf{j}]^{4}\right)+ \\
& +\varepsilon \mathbf{X}[2]\left(\mathbf{T}[2]^{4}-\mathrm{T}_{\infty}^{4}\right)=\mathbf{Q}_{\text {int }}[2]+\alpha \mathbf{X}[2] \mathbf{q}_{\text {ext }}[2]+\mathbf{X}[6] \\
& \sum_{\mathbf{j}=1}^{6} \mathbf{G}_{3, \mathrm{j}}(\mathbf{T}[3]-\mathbf{T}[\mathbf{j}])+\sum_{\mathrm{j}=1}^{6} \mathbf{R}_{3, \mathrm{j}}\left(\mathbf{T}[3]^{4}-\mathbf{T}[\mathbf{j}]^{4}\right)+ \\
& +\varepsilon \mathbf{X}[3]\left(\mathbf{T}[3]^{4}-\mathrm{T}_{\infty}^{4}\right)=\mathbf{Q}_{\text {int }}[3]+\alpha \mathbf{X}[3] \mathbf{q}_{\text {ext }}[3] \\
& \sum_{\mathrm{j}=1}^{6} \mathbf{G}_{4, \mathrm{j}}(\mathbf{T}[4]-\mathbf{T}[\mathbf{j}])+\sum_{\mathrm{j}=1}^{6} \mathbf{R}_{4, \mathrm{j}}\left(\mathbf{T}[4]^{4}-\mathbf{T}[\mathbf{j}]^{4}\right)+ \\
& +\varepsilon \mathbf{X}[4]\left(\mathbf{T}[4]^{4}-\mathrm{T}_{\infty}^{4}\right)=\mathbf{Q}_{\text {int }}[4]+\alpha \mathbf{X}[4] \mathbf{q}_{\text {ext }}[4] \\
& \sum_{\mathrm{j}=1}^{6} \mathbf{G}_{5, \mathrm{j}}(\mathbf{T}[5]-\mathbf{T}[\mathbf{j}])+\sum_{\mathrm{j}=1}^{6} \mathbf{R}_{5, \mathrm{j}}\left(\mathbf{T}[5]^{4}-\mathbf{T}[\mathbf{j}]^{4}\right)+ \\
& +\varepsilon \mathbf{X}[5]\left(\mathbf{T}[5]^{4}-\mathrm{T}_{\infty}^{4}\right)=\mathbf{Q}_{\text {int }}[5]+\alpha \mathbf{X}[5] \mathbf{q}_{\text {ext }}[5] \\
& \sum_{j=1}^{6} \mathbf{G}_{6, j}(\mathbf{T}[6]-\mathbf{T}[\mathbf{j}])+\sum_{j=1}^{6} \mathbf{R}_{6, j}\left(\mathbf{T}[6]^{4}-\mathbf{T}[\mathbf{j}]^{4}\right)=0
\end{aligned}
$$

For a given $\mathbf{X}$, the solution of the system of algebraic equations (1) to (6) results in two set of temperatures: $\mathbf{T}_{\mathbf{C C}}(\mathbf{X})$ [1:6] if $\mathrm{CC}$ conditions were applied (minimum values of $\mathbf{Q}_{\text {int }}, \mathbf{q}_{\text {ext }}$ and $\alpha$ ) and $\mathbf{T}_{\mathbf{H C}}(\mathbf{X})[1: 6]$ if HC conditions were applied (maximum values of of $\mathbf{Q}_{\text {int }}, \mathbf{q}_{\text {ext }}$ and $\alpha$ ). The coefficients of the equations (1) to (6) and the solution of the system of equation are obtained by mean of INPE PCTER thermal software package ${ }^{[20]}$, which was coupled to the optimization algorithm.

Constraints are posed to the panels temperatures, which must lie inside required design intervals. The target temperature and allowed range for each panel are defined as a function of the thermal requirements of the equipment mounted on the respective panel.

Mathematically, the multiobjective problem of minimizing the differences between the target and the operational temperatures for both $\mathrm{CC}$ and $\mathrm{HC}$, and of minimizing the battery heater power is formulated as a mono-objective optimization problem, assuming unitary weighting factors ${ }^{[16]}$ before each term of the objective function:

$$
\begin{aligned}
\text { Minimize } \mathrm{F}(\mathbf{X})=\left\|\mathbf{T}_{\mathbf{C C}}(\mathbf{X})-\mathbf{T}_{\mathrm{T}}\right\|_{2} \\
+\left\|\mathbf{T}_{\mathbf{H C}}(\mathbf{X})-\mathbf{T}_{\mathrm{T}}\right\|_{2}+\mathbf{X}[6] \\
\text { Subj. to: } \mathbf{X}_{\mathrm{MIN}} \leq \mathbf{X} \leq \mathbf{X}_{\mathrm{MAX}} \\
\mathbf{T}_{\mathrm{MIN}} \leq \mathbf{T}_{\mathbf{C C}}(\mathbf{X}) \leq \mathbf{T}_{\mathrm{MAX}} \\
\mathbf{T}_{\mathrm{MIN}} \leq \mathbf{T}_{\mathbf{H C}}(\mathbf{X}) \leq \mathbf{T}_{\mathbf{M A X}}
\end{aligned}
$$


Hereafter, the complete optimization problem will be referred to as the Cold and Hot Case (CHC).

Additionally, the $\mathrm{CC}$ and the $\mathrm{HC}$ are formulated and solved as optimization problems separately. The idea is to get some insight by comparing these separated $\mathrm{CC}$ and $\mathrm{HC}$ solutions with the combined Cold and Hot Case (CHC) solutions, defined in equation 7. Mathematically, the $\mathrm{CC}$ and $\mathrm{HC}$ optimization problems are stated as follows.

\section{Cold Case (CC):}

$$
\begin{aligned}
& \text { Min. } \begin{aligned}
F(X)=\operatorname{Min}\left\|\mathbf{T}_{\mathbf{C C}}(\mathbf{X})-\mathbf{T}_{\mathbf{T}}\right\|_{2} \\
\text { Subj. to: } \mathbf{X}_{\mathbf{M I N}} \leq \mathbf{X} \leq \mathbf{X}_{\mathbf{M A X}} \\
\mathbf{T}_{\mathbf{M I N}} \leq \mathbf{T}_{\mathbf{C C}}(\mathbf{X}) \leq \mathbf{T}_{\mathbf{M A X}}
\end{aligned}
\end{aligned}
$$

\section{Hot Case (HC):}

$$
\begin{aligned}
& \text { Min. } \begin{aligned}
F(X)=\operatorname{Min}\left\|\mathbf{T}_{\mathbf{H C}}(\mathbf{X})-\mathbf{T}_{\mathbf{T}}\right\|_{2} \\
\text { Subj. to: } \mathbf{X}_{\mathbf{M I N}} \leq \mathbf{X} \leq \mathbf{X}_{\mathbf{M A X}} \\
\mathbf{T}_{\mathbf{M I N}} \leq \mathbf{T}_{\mathbf{H C}}(\mathbf{X}) \leq \mathbf{T}_{\mathbf{M A X}}
\end{aligned}
\end{aligned}
$$

It is important to notice that in the $\mathrm{CC}$ and $\mathrm{HC}$ above, $\mathbf{X}=\mathbf{X}[1: 5]$ = area of radiators on panels 1 to 5 , respectively (there is no $\mathrm{X}[6]$ ). Besides that, there is no battery heater being used in the $\mathrm{CC}$ and $\mathrm{HC}$ stand-alone cases.

\section{RESULTS}

Table 2 summarizes the limits on the design variables, the operational temperature limits, as well as the internal heat dissipation from the electronic devices applied to the panels.

Table 2 - Design variable limits, operational limits and panel heat dissipation.

\begin{tabular}{|c|c|c|c|c|c|c|c|}
\hline \multirow{2}{*}{ Parameter } & \multicolumn{7}{|c|}{ Panel } \\
\cline { 2 - 7 } \multicolumn{2}{|c|}{} & 1 & 2 & 3 & 4 & 5 & $6^{1)}$ \\
\hline $\begin{array}{c}\text { Radiator } \\
\text { area limits } \\
\left(\mathrm{m}^{2}\right)^{2}\end{array}$ & $\mathrm{Xmin}$ & 0.0 & 0.0 & 0.0 & 0.0 & 0.0 & - \\
\cline { 2 - 8 } & $\mathrm{Xmax}$ & 0.902 & 0.952 & 0.952 & 0.952 & 0.952 & - \\
\hline $\begin{array}{c}\text { Temperature } \\
\text { limits }\left({ }^{\circ} \mathrm{C}\right)\end{array}$ & $\mathrm{Tmin}$ & -5.0 & -10.0 & -20.0 & -20.0 & -10.0 & -20.0 \\
\cline { 2 - 8 } & $\mathrm{Tmax}$ & +50.0 & +20.0 & +50.0 & +45.0 & +45.0 & +50.0 \\
\hline $\begin{array}{c}\text { Target } \\
\text { temperature }\end{array}$ & $\begin{array}{c}\mathrm{T}_{\mathrm{T}} \\
\left({ }^{\circ} \mathrm{C}\right)\end{array}$ & +22.5 & +15.0 & $+15.0+12.5$ & +17.5 & +15.0 \\
\hline $\begin{array}{c}\text { Internal } \\
\text { heat } \\
\text { dissipation } \\
(\mathrm{W})\end{array}$ & $\mathrm{CC}$ & 15.0 & $13.0^{5}$ & 8.6 & 40.0 & 20.0 & 0.0 \\
\cline { 2 - 8 } & $\mathrm{HC}$ & 40.0 & 47.5 & 27.2 & 55.0 & 90.5 & 0.0 \\
\hline
\end{tabular}

${ }^{1)}$ Top panel of the PMM, thermally isolated from the payload. ${ }^{2)}$ The $6^{\text {th }}$ element of this parameter exists only for $\mathrm{CHC}$ and is not a radiator area. It represents the heater power on panel 2 for the $\mathrm{CHC}$, with the following limits: $\mathrm{X}_{\mathrm{MIN}}[6]=0 \mathrm{~W}$ and $\mathrm{X}_{\mathrm{MAX}}[6]=65 \mathrm{~W}$. ${ }^{3)}$ The temperature limits of each panel were determined according to the most restrictive equipment mounted on it. ${ }^{4)}$ The internal heat dissipation of each panel is the summation of the heat dissipation of all equipment mounted on the respective panel. ${ }^{5)}$ For the $\mathrm{CHC}$, in addition to the minimum heat dissipation of the equipment on this panel, the battery heater dissipates in the range $0.0 \leq \mathrm{X}[6] \leq 65.0 \mathrm{~W}$.

Each design variable was encoded in 7 bits, what means a resolution better than $0.01 \mathrm{~m}^{2}$ and $1.0 \mathrm{~W}$ for the radiator areas and the heater dissipation, respectively.

The search for optimal designs was made for different values of $\tau$ within the range $[0.0,5.0]$, using a 0.5 step size, in order to find $\tau=\tau^{*}$. As the problem is a computationally costly one, the number of function evaluations (NFE) for each $\tau$ was set to $5 \times 10^{3}$ and an average of 25 independent runs was taken. A more detailed description is given below. The following steps were performed (here, GEO means GEO/ $\mathrm{GEO}_{\text {var }}$ ):

a) Run GEO with $5 \times 10^{3}$ function evaluations, 25 independent runs (25 different seeds) for each $\tau$ of the set $\{0.0,0.5,1.0,1.5,2.0,2.5,3.0,3.5,4.0,4.5,5.0]$;

b) For each $\tau$ in a), calculate the average value of the $25 \mathrm{~F}$ (Xbest) found, where Xbest = best solution found after $5 \times 10^{3}$ function evaluations. Three different procedures were used to calculate the average, as follow (so, three different averages were obtained):

b.1) From the 25 runs, calculate the average using only those where a feasible solution occured;

b.2) Calculate the average using all 25 runs. For the infeasible solutions, use the value of the worst feasible solution found;

b.3) From the 25 runs, get the best F(Xbest) found as the average.

c) Plot the three curves "average F(Xbest) versus $\tau$ ";

d)For each curve in c), locate the minimum;

e) Calculate the average of the three values obtained in d) and use it as the $\tau^{*}$.

The Table 3 shows the $\tau=\tau^{*}$ calculated as explained above.

Table $3-\tau^{*}$ found for $\mathrm{CC}, \mathrm{HC}$ and $\mathrm{CHC}$.

\begin{tabular}{|c|c|c|c|c|c|c|}
\hline Case & \multicolumn{2}{|c|}{$\mathrm{CC}$} & \multicolumn{2}{c|}{$\mathrm{HC}$} & \multicolumn{2}{c|}{ CHC } \\
\hline Algorithm & GEO & GEO $_{v a}$ & GEO & GEO $_{v a}$ & GEO & GEO $_{v a}$ \\
\hline$\tau^{*}$ & 2.7 & 3.2 & 1.3 & 1.3 & 2.0 & 2.3 \\
\hline
\end{tabular}

After that, the $\tau^{*}$ values were used and 25 runs were performed for each case, each of them stopped 
after $10^{5}$ function evaluations. The best designs found with GEO and $\mathrm{GEO}_{\text {var }}$ for each case are in Table 4.

Table 4 - Best designs for CC, $\mathrm{HC}$ and $\mathrm{CHC}$.

\begin{tabular}{|c|c|c|c|c|c|c|c|c|}
\hline Case & $\begin{array}{c}\text { Algorit } \\
\text { hm }\end{array}$ & $F(X)$ & $X[1]$ & $X[2]$ & $X[3]$ & $X[4]$ & $X[5]$ & $X[6]$ \\
\hline \multirow{2}{*}{ CC } & GEO & 4.018 & 0.001 & 0.062 & 0.048 & 0.251 & 0.030 & - \\
\cline { 2 - 9 } & GEOvar & 4.360 & 0.008 & 0.062 & 0.035 & 0.271 & 0.022 & - \\
\hline \multirow{2}{*}{ HC } & GEO & 1.487 & 0.028 & 0.433 & 0.143 & 0.359 & 0.457 & - \\
\cline { 2 - 8 } & GEOvar & 1.789 & 0.028 & 0.433 & 0.129 & 0.372 & 0.457 & - \\
\hline \multirow{2}{*}{ CHC } & GEO & 142.1 & 0.838 & 0.433 & 0.055 & 0.042 & 0.044 & 57.3 \\
\hline
\end{tabular}

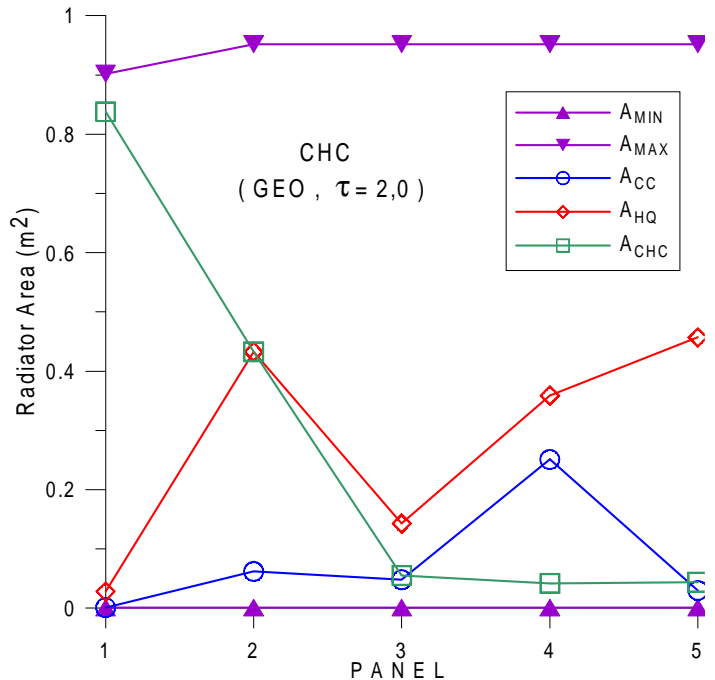

Figure 4 - Radiator areas for CC, $\mathrm{HC}$ and CHC (GEO)

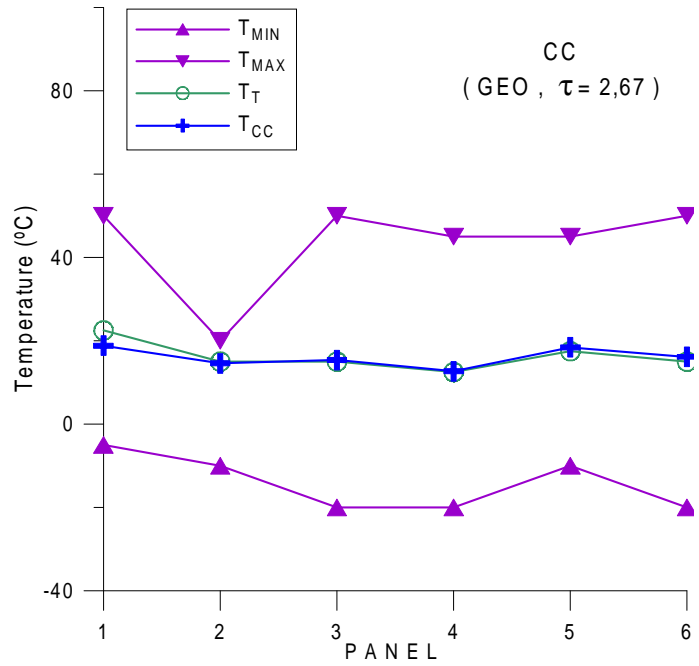

Figure 6 - Resulting temperatures for CC (GEO)
From the results shown on Table 4 it can be seen that both GEO and GEO $_{\text {var }}$ have found very close design solutions for $\mathrm{CC}$ and $\mathrm{HC}$. For $\mathrm{CHC}$, the design solutions differ considerably, but both have very close $\mathrm{F}(\mathbf{X})$ values, meaning, possibly, the existence of several quasi-optimal solutions.

Figures 4 and 5 present, for GEO and $\mathrm{GEO}_{\text {var }}$, respectively, the radiator areas obtained for $\mathrm{CC}, \mathrm{HC}$ and CHC. The lower and upper limits for the radiator area of each panel are also presented. Analyzing these figures, it is possible to conclude that an intuitive solution based on a linear interpolation between the $\mathrm{CC}$ and $\mathrm{HC}$ solutions would not lead to the solution obtained for CHC.

The temperatures on the panels, calculated with the data of Table 2 are shown on Figures 6 to 11.

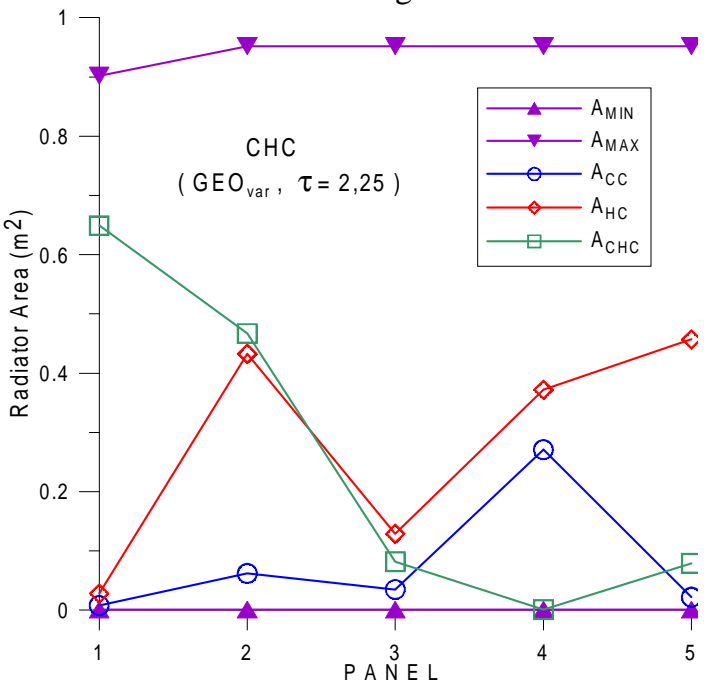

Figure 5 - Radiator areas for $\mathrm{CC}, \mathrm{HC}$ and $\mathrm{CHC}\left(\mathrm{GEO}_{\mathrm{vax}}\right)$

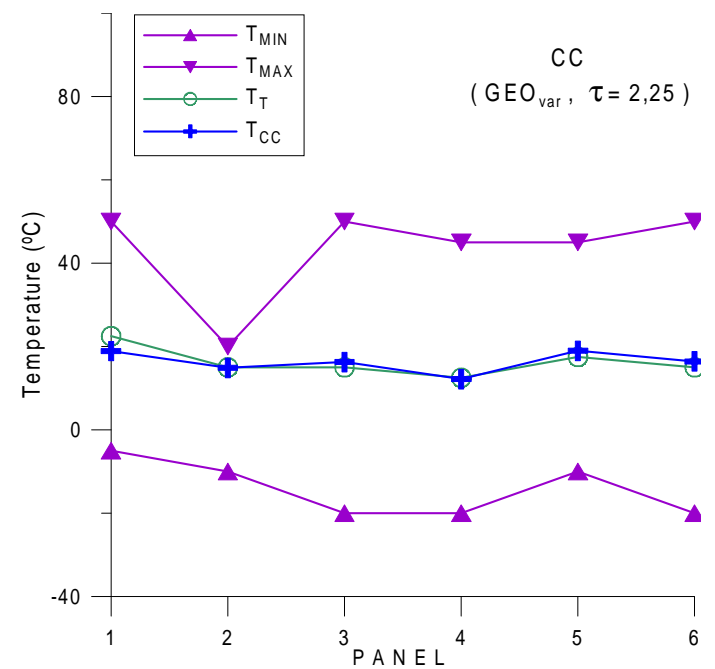

Figure 7 - Resulting temperatures for $\mathrm{CC}\left(\mathrm{GEO}_{\mathrm{vad}}\right)$ 


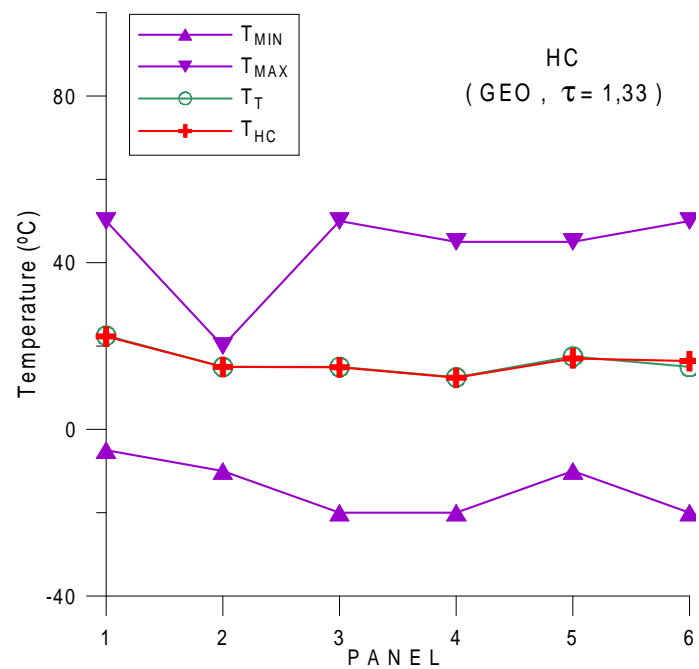

Figure 8 - Resulting temperatures for $\mathrm{HC}$ (GEO)

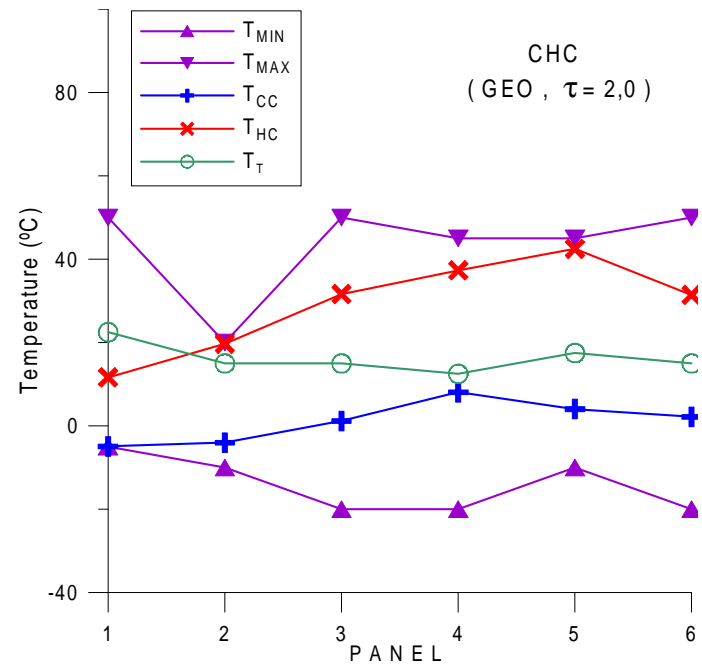

Figure 10 - Resulting temperatures for $\mathrm{CHC}$ (GEO)

What can be readily seen is that, for CC and $\mathrm{HC}$, the optimized design solutions brought the resulting panels temperatures very close to the target temperatures. That is not the case for $\mathrm{CHC}$, where most panels temperatures are far from the target temperatures. In fact, some of them are at the limit temperatures of the respective panel. Despite of having different values on the design variables, both $\mathrm{CHC}$ solutions had the panels 1,2 and 5 as those where temperatures are at the limit.

Another important observation is that, though being numerically quite close in the $\mathrm{F}(\mathrm{X})$ value, from an engineering point of view, the $\mathrm{GEO}_{\text {var }}$ solution for $\mathrm{CHC}$ is quite different from the GEO solution. The first needs only $49.7 \mathrm{~W}$ for the heater power (X[6]), against $57.3 \mathrm{~W}$ of the second. Remembering that $\mathrm{X}[6]$ is the third term

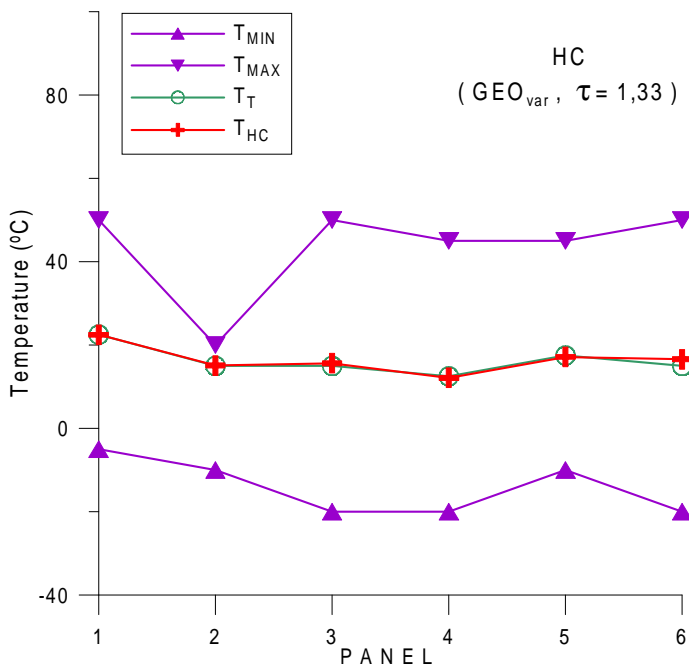

Figure 9 - Resulting temperatures for $\mathrm{HC}\left(\mathrm{GEO}_{\mathrm{var}}\right)$

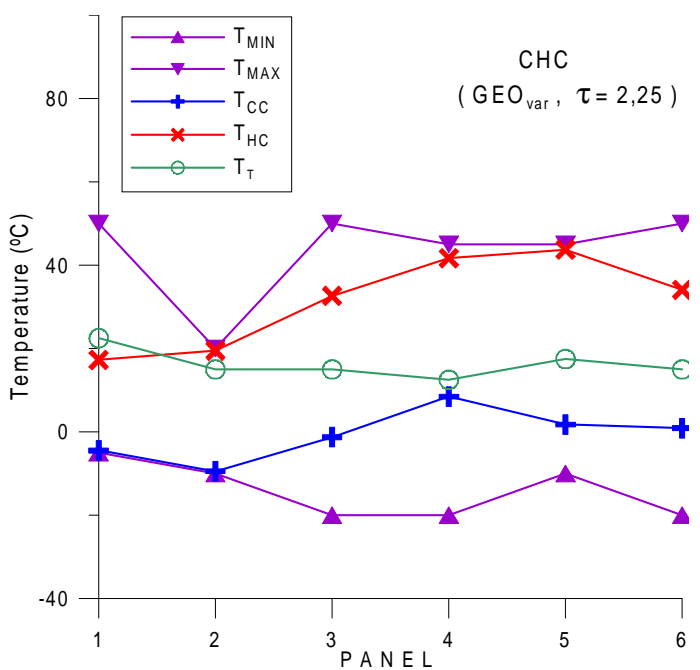

Figure 11 - Resulting temperatures for $\mathrm{CHC}\left(\mathrm{GEO}_{\text {var }}\right)$

of $\mathrm{F}(\mathrm{X})$ and, as both solutions have almost the same $F(X)$, the counterpart is that the $49.7 \mathrm{~W}$ solution has a worst performance on the first two terms (temperature part) of $\mathrm{F}(\mathrm{X})$, in order to reestablish the equality. In our opinion, the main point is the possibility of choice between these two equivalent solutions. The designer can possibly decide which one is better by considering other quantifiers, not present in the formulation of $F(X)$, just because they were not thought about or even because they were too difficult to quantify. Examples of such quantifiers are assembly easiness, durability, and robustness, just to mention a few. This possibility only exists if one has some knowledge about the existence of such equivalent optimal solutions. 


\section{CONCLUSION AND FUTURE DIRECTIONS}

In this paper the inverse design of the thermal control system of the PMM spacecraft was done successfully. The design solutions found were nonintuitive ones, unlikely to occur as possibilities for a human designer. The optimizing procedure chosen, the GEO algorithm, has shown to be a valuable optimal design tool, being not only easy to implement but also, easy to have its $\tau$ parameter set to the particular application tackled. The application itself has proven to be a very restrictive one, even when the $\mathrm{HC}$ and $\mathrm{CC}$ cases were considered alone. The unfeasible design space was considerably large. However, this posed no difficulties on the GEO functioning, as expected. Based on the two design solutions found for $\mathrm{CHC}$, and on the considerations already made about them, it seems to be valuable as a future development to formulate and solve the $\mathrm{CHC}$ as a multiobjective problem. For instance, we may define the sum of the first two terms of the $F(X)$ equation for $\mathrm{CHC}$ as one objective $\left(\mathrm{f}_{1}\right)$ and the heater power as the second objective $\left(f_{2}\right)$. In this case, the Pareto frontier is a curve on the $\mathrm{f}_{1} \mathrm{xf}_{2}$ objective space where each point of the curve establishes an optimal compromise between these two objectives. This allows the designer to look at the whole set of such solutions and choose the most appropriated one. In fact, the two solutions given by GEO and $\mathrm{GEO}_{\text {var }}$ for $\mathrm{CHC}$ belong to the Pareto frontier.

\section{REFERENCES}

1. F. L. Sousa and F. M. Ramos Function Optimization Using Extremal Dynamics, Proceedings of the 4th International Conference on Inverse Problems in Engineering (cd-rom), Rio de Janeiro, Brazil, 2002.

2. F. L. Sousa, F. M. Ramos, P. Paglione and R. M. Girardi New stochastic algorithm for design optimization. AIAA Journal, Vol. 41, Number 9, pp. 1808-1818, 2003.

3. F. L. Sousa, F. M. Ramos, R. L. Galski and I. Muraoka "Generalized Extremal Optimization: A New Meta-heuristic Inspired by a Model of Natural Evolution". Accepted for publication in Recent Developments in Biologically Inspired Computing, De Castro, L. N. \& Von Zuben, F. J. (editors), Idea Group Inc., 2004.

4. P. Bak and K. Sneppen Punctuated equilibrium and criticality in a simple model of evolution. Physical Review Letters, v. 71, n. 24, p. 4083-4086, 1993.

5. F. L. Sousa; V. Vlassov and F. M. Ramos Heat pipe design through generalized extremal optimization. Proceedings of the IX Brazilian Congress of
Engineering and Thermal Sciences - ENCIT 2002, Caxambu, MG, Brazil, 2002.

6. G. N. Vanderplaats Numerical optimization techniques for engineering design. Colorado Springs: Vanderplaats Research \& Development, 2 edition, 1998.

7. P. M. Pardalos and H. E. Romeijn (Editors). Handbook of global optimization. Vol. 2, Kluwer Academic Publishers, Norwell, MA, 2002.

8. F. W. Glover and G. A. Kochenberg (Editors), Handbook of Metaheuristics. International Series in Operations Research \& Management Science, Vol. 57, Kluwer Academic Publishers, Boston, 2003, 570 pp.

9. M. S. Eldred Optimization strategies for complex engineering applications. SANDIA Report, SAND98-0340, UC-705, 1998.

10. D. E. Goldberg Genetic Algorithms in Search, Optimization, and Machine Learning, Addison-Wesley Publishing Company, 1989.

11. L. D. Davis, K. De Jong, M. D. Vose, L. D. Whitley, (Editors). Evolutionary algorithms. The IMA Volumes in Mathematics and its Applications, Vol. 111, Springer-Verlag, 1999.

12. S. Kirkpatrick, C. D. Gellat and M. P. Vecchi Optimization by Simulated Annealing, Science, Vol. 220, Number 4598, 1983, pp. 671-680.

13. J. A. Freeman, I. Skapura Neural networks: algorithms, applications and programming techniques. New York: Addison-Wesley, 1991.

14. L. N. Castro and J. Timmis An Artificial Immune Network for Multimodal Function Optimization. Proceedings of the IEEE Congress on Evolutionary Computation (CEC'02), Vol. 1, pp. 699-674, May, Hawaii, 2002.

15. E. Bonabeau, M. Dorigoú, G. Theraulaz Inspiration for Optimization From Social Insect Behaviour. Nature, v. 406, p. 39-42, 2000.

16. K. Miettinen Some Methods for Nonlinear Multi-objective Optimization, Lecture Notes in Computer Science: Evolutionary MultiCriterion Optimization, v1993, 1-20, 2001.

17. V. Pareto Manuale di economia politica com una introduzione alla scienza sociele, Milan: Società Editrice Libraria, 1909.

18. P. Bak How nature works. New York: Copernicus, Springer-Verlag, 1996.

19. D. G. Gilmore Satellite Thermal Control Handbook. The Aerospace Corporation Press, El Segundo, CA, 1994.

20. H.P. Cardoso, I. Muraoka, J.L.F. Bastos, L.A.W. Bambace, O.B. Oliveira Filho and R.M.G. Leite PCTER Thermal Analysis Software, User's Manual (In Portuguese), INPE, São José dos Campos, SP, Brazil, 1990. 